\title{
Revisiting Lunar Water Ice Content Retrieval Using Lunar Remote Sensing Data
}

\author{
Vaidehi Patel ${ }^{1}$,Jayesh Pabari ${ }^{2}$, Jignesh Patel $^{3}$ \\ ${ }^{1}$ E\&C Dept. SVIT, Vasad, India \\ ${ }^{2}$ Physical Research Laboratory, Ahmedabad, India \\ ${ }^{3}$ E\&C Dept. SVIT, Vasad, India
}

\begin{abstract}
During a few recent lunar missions, a possibility of water ice existing in the permanently shadowed regions on the moon has come forward. The content of water ice on the moon is an open question in the current moon study. It is known that the existence of ice mixed with regolith can affect permittivity of the lunar surface material and consequently to the microwave brightness temperature of the lunar surface. In this paper, we have considered the measured permittivity of the lunar soil simulant JSC-1A and the pure ice. A few dielectric mixing models have been discussed and Odelevsky model has been used to derive the effective permittivity of the mixture. The microwave brightness temperature has been simulated using two layer lunar regolith model. The relationship between the microwave brightness temperature and the water ice content has been arrived at using the derived effective permittivity. The microwave brightness temperature of the lunar surface obtained by Changé-1 has been used. From the study, it has been found that there is a possibility of upper regolith layer with about $9 \mathrm{~cm}$ can have the volume water ice content of about $2.943 \%$.
\end{abstract}

Key Words: Brightness Temperature, Microwave, Moon, Permittivity, Regolith, Water Ice

\section{Motivation}

Moon is the only natural satellite of the earth. Interest in moon increased as a result of various successful missions to the Moon like Selene (Japan), Chang'e-1 and Chang'e-2 (China), LRO (USA), Chandrayaan-1(India). Detection of presence of water ice within the planetary regolith is crucial for future manned explorations as well as for understanding geological history of the surface. Moon is known for having either no atmosphere or very light atmosphere, where no water can survive in its free form. The possibility of water/ice on the Moon could be due to bombardment of comets on lunar surface. The water molecules could be trapped inside the permanently dark regions near the pole. Thus, water can be present in the form of ice or mixed with lunar soil (regolith). Water ice content may be determined on the basis of theoretical modeling or using various detections methods sensitive to the water ice. This is the motivating factor for us to revisit the retrieval of water ice content on the moon using theoretical modeling.

\section{Introduction}

There has been a controversy on the presence of water ice on the Moon. Arnold was the first to suggest possible sources of the water ice near the lunar poles [1]. Watson and Arnold found that the most of the lunar polar regions are in the permanent shadow and the temperature at those locations is sufficiently low for the water to be preserved in the form of ice [2]. Clementine orbiter showed that the water might be present from the radar based experiment [3], but a later on Spudis [4] showed on the basis of earth based Arecibo observatory that water might not be present. Lunar Prospector (LP) neutron data, $\gamma$ spectrometer data and radar data [5] found that water is present in the form of ice at lunar poles but a reanalysis by Nozettel and Hodges found that it was not but they found that Hydrogen is present based on theoretical modeling [3],[6]. The LRO mission then verified the existence of water ice on the moon [5],[6]. Using Clementine UVVIS data and improved Dobson model, Meng [7] evaluated water ice content near the poles and found water ice content to be $1.73 \%$. Meng, Chen and his colleagues [8] used Odelevsky dielectric mixing model and Chang'e-1 data to obtain water ice content in the Cabeus crater near the south pole and they found it to be $2.83 \%$. To obtain water ice content, microwave radiometer data are feasible as the previous researches [8] indicate. Therefore, in this paper, the passive microwave data from microwave radiometer onboard Chang'e-1 satellite are used and microwave radiative transfer equation is used based on the regolith model. The regolith in Cabeus crater could be the mixture of regolith and the water ice. The dielectric mixing models are compared to establish the relationship between the volume water ice content and the effective dielectric constant. Then, volume water ice content is obtained based on the improved radiative transfer model, the selected dielectric mixing model and the lunar microwave brightness temperature data. 


\section{Lunar Regolith Model}

The lunar regolith model generally used is a three layer model in which the top dust, lunar regolith and bed rock layers are considered. Chen and his colleagues [8] have used single layer model i.e. mixture of regolith with the water ice. The brightness temperature of the lunar regolith layer in the passive microwave remote sensing is numerically simulated by a three-layer model (the layering dust, regolith, and underlying rock media) using fluctuation dissipation theorem [9]. Based on the study by Shkuratov [10], Jin [11], Meng [12] and Wang [13], the complete lunar regolith model should consider the temperature profile, dielectric constant distribution, effective surface reflectivity.

As the penetration depth of microwave is lesser at higher frequency, one can ignore the contribution from the bottom layers. Therefore, in this paper, two layer model is considered for retrieval of the water ice content. The first layer is a regolith mixed with the ice and the second layer is only regolith. Fig.1 [9] show the lunar regolith model used in this study. The model also shows the brightness temperature emissions from both the layers. The depth of the first layer is $d_{1} m$ and that of the second layer is $d_{2} m$.

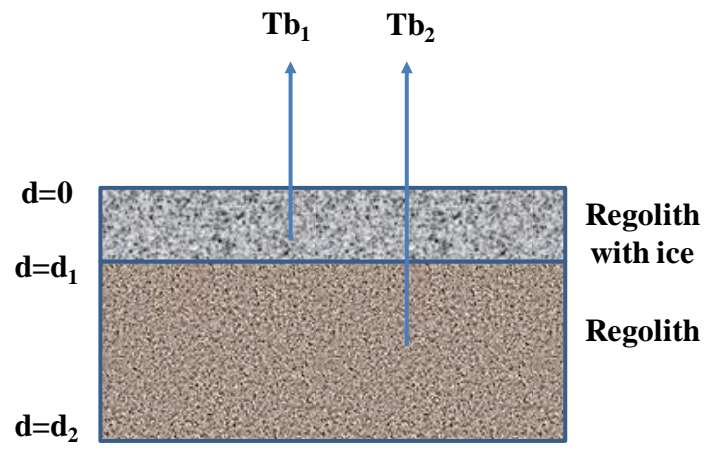

Figure 1: Lunar regolith model

Based on this two layer regolith model, the brightness temperature is given by [9]

$\mathrm{T}_{\mathrm{B}}=\left(1-\mathrm{r}_{01}\right)\left(1-\mathrm{e}^{-\alpha_{1} \vartheta \mathrm{d}_{1}}\right)\left(1+\mathrm{r}_{12} \mathrm{e}^{-\alpha_{1} \vartheta \mathrm{d}_{1}}\right) \mathrm{T}_{1}+\left(1-\mathrm{r}_{01}\right)\left(1-\mathrm{r}_{12}\right)\left(1-\mathrm{e}^{-\alpha_{1} \vartheta \mathrm{d}_{2}}\right) \mathrm{e}^{-\alpha_{1} \vartheta \mathrm{d}_{1}} \mathrm{~T}_{2}+\mathrm{T}_{\mathrm{BG}}$

where,

$T_{1}$ and $T_{2}$ are physical surface and deep temperatures, respectively,

$r_{01}$ is the effective reflectivity of free space and the regolith (with ice) interface

$r_{12}$ is effective reflectivity of the regolith (with ice) and the regolith interface.

The parameters are functions of the dielectric constants of the free space, the regolith with water ice and the regolith.

$\vartheta$ is the frequency;

$\mathrm{T}_{\mathrm{BG}}$ is the cosmic background temperature.

$\alpha_{1}$ and $\alpha_{2}$ are the attenuation factors of the regolith with water ice and the only regolith;

$\alpha_{i}=\frac{2 \pi \times \varepsilon_{i}^{\prime \prime}}{c \sqrt{\varepsilon_{i}^{\prime}}}$

where

$\varepsilon_{i}^{\prime}$ is the real part of dielectric permittivity,

$\varepsilon_{i}$ " is the imaginary part of dielectric permittivity and

$\mathrm{c}$ is the velocity of light. 


\section{Permittivity of the Regolith with Water Ice}

There is a possibility of water ice present in the permanently shaded regions of the Moon as indicated by NASA [14]. In this paper, regolith with water ice mixture is considered as made of the dry lunar regolith and the pure ice. The dielectric mixing models may be used to evaluate the effective permittivity of the mixture. Various dielectric mixing models used [15] are

1. Refractive model

2. Wagner \& landau-lifshtiz model

3. Clausis-Mosotti,Lorenz-Lorentz model

4. Litchenecker model

5. Odelevsky model

The equations of various mixing models are as follows

1. Refractive model

$\sqrt{\varepsilon_{\text {eff }}}=W_{1} \sqrt{\varepsilon_{\mathrm{w}}}+\mathrm{W}_{2} \sqrt{\varepsilon_{\mathrm{s}}}$

where

$\varepsilon_{\text {eff }}=$ dielectric constant of dense medium

$\varepsilon_{\mathrm{w}}=$ dielectric constant of water

$\varepsilon_{\mathrm{s}}=$ dielectric constant of regolith

$\mathrm{W}_{1}=$ Volumetric water content, $\%$

$\mathrm{W}_{2}=$ Regolith content, $\%$

2. Wagner \& landau-lifshtiz model

$\varepsilon_{\text {eff }}=\varepsilon_{\mathrm{s}}\left(1+3 \mathrm{~W} \frac{\varepsilon_{\mathrm{s}}-\varepsilon_{\mathrm{w}}}{\varepsilon_{\mathrm{s}}+2 \varepsilon_{\mathrm{w}}}\right)$

where

$\varepsilon_{\text {eff }}=$ dielectric constant of dense medium

$\varepsilon_{\mathrm{w}}=$ dielectric constant of water

$\varepsilon_{\mathrm{s}}=$ dielectric constant of regolith

$\mathrm{W}=$ Volumetric water content, $\%$

3. Clausis-Mosotti,Lorenz-Lorentz model

$\varepsilon_{\text {eff }}=\varepsilon_{\mathrm{w}}\left[1+\frac{\mathrm{w}}{\frac{1-\mathrm{w}}{3}+\frac{\varepsilon_{\mathrm{w}}}{\varepsilon_{\mathrm{s}}-\varepsilon_{\mathrm{w}}}}\right]$

where

$\varepsilon_{\text {eff }}=$ dielectric constant of dense medium

$\varepsilon_{\mathrm{w}}=$ dielectric constant of water

$\varepsilon_{\mathrm{s}}=$ dielectric constant of regolith

$\mathrm{W}=$ Volumetric water content, $\%$

4. Litchenecker model

$\log \varepsilon_{\text {eff }}=W_{1} \log \varepsilon_{w}+W_{2} \log \varepsilon_{s}$

where

$\varepsilon_{\text {eff }}=$ dielectric constant of dense medium

$\varepsilon_{\mathrm{w}}=$ dielectric constant of water

$\varepsilon_{\mathrm{s}}=$ dielectric constant of regolith

$\mathrm{W}_{1}=$ Volumetric water content, $\%$

$\mathrm{W}_{2}=$ Regolith content, $\%$

5. Odelevsky model

$\varepsilon_{\mathrm{eff}}=\varepsilon_{\mathrm{w}}\left[1+\frac{1-\mathrm{w}}{\frac{\mathrm{w}}{3}+\frac{\varepsilon_{\mathrm{w}}}{\varepsilon_{\mathrm{s}}-\varepsilon_{\mathrm{w}}}}\right]$

where 
$\varepsilon_{\text {eff }}=$ dielectric constant of dense medium

$\varepsilon_{\mathrm{w}}=$ dielectric constant of water

$\varepsilon_{\mathrm{s}}=$ dielectric constant of regolith

$\mathrm{W}=$ Volumetric water content, \%

In the Cabeus crater, there are permanently shaded regions where the temperature is about $50 \mathrm{~K}$ [16]. The dielectric constant is affected only by the permittivity of the regolith and that of the water ice. We have considered the permittivity of the lunar soil stimulant JSC-1A as $\varepsilon_{s}=2.67+8.064 E-07 i$ and permittivity of the pure ice is taken as $\varepsilon_{i}=4.01+6.53 i$ [17]. Various mixing models have been used to estimate the effective permittivity of the mixture, which is considered for the Cabeus crater. The change of effective permittivity in Cabeus crater with volume water ice content as estimated from the five mixing models are shown in Fig. 2.
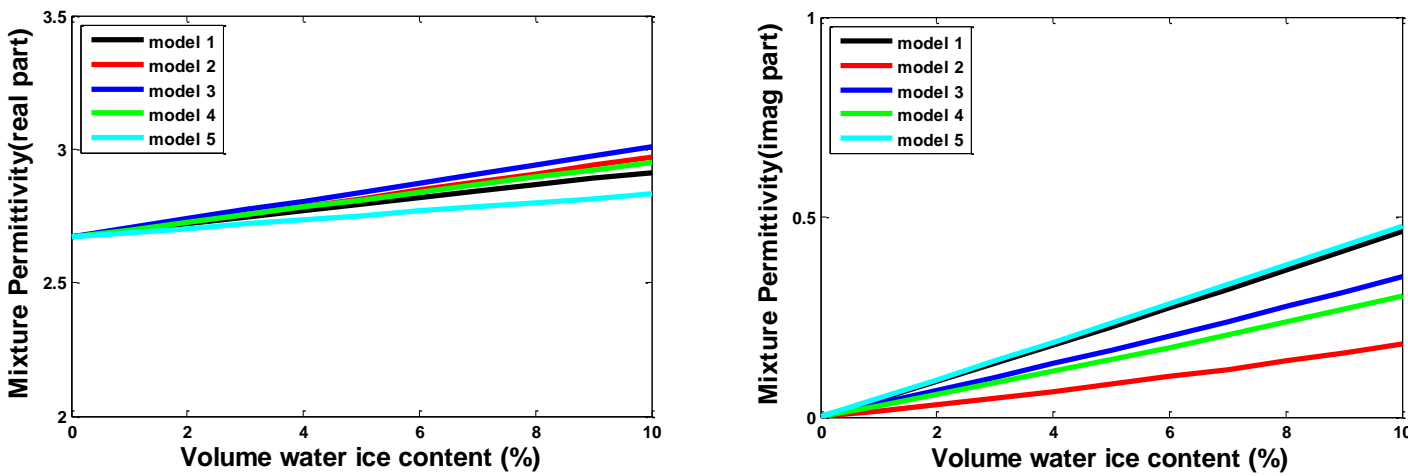

Figure 2: Variation of (a) real part (top) and (b) imaginary part (bottom) of permittivity with respect to the volume ice content.

Fig. 2 depicts that the permittivity increases with the given volume ice content. The Odelevsky model is better than the other four models [8]. Therefore, in this study, Odelevsky model is used to obtain dielectric constant of the Cabeus crater.

\section{Water Ice Content Retrieval for Cabeus Crater}

Cabeus crater is located near lunar south pole, ranging from $30^{\circ} \mathrm{W}$ to $60^{\circ} \mathrm{W}$ in longitude and from $84^{\circ} \mathrm{S}$ to $86^{\circ} \mathrm{S}$ in latitude [7] which is in a permanently shadowed region. China's first lunar orbiter, Chang'e-1 had four-channel microwave radiometer. The four frequencies of the radiometer are 3.0, 7.8, 19.35 and $37 \mathrm{GHz}$. The Microwave Radiometer (MRM) on board Change-1 mission is the first passive microwave remote sensor and provides eight time coverage to measure microwave brightness temperature of the moon. The MRM data are superior to other ground based observation in terms of spatial resolution, temperature sensitivity and coverage area [8]. It is known that the shorter wavelength of the passive microwave signal can penetrate the lunar regolith only up to few centimeters to meters.

The selection of the study point is critical. In permanently shadowed region, the temperature is very low and corresponding temperature profile of lunar regolith is steady [8]. Selection criteria has been given by [8] as brightness temperature to be very low and the difference between values observed in several time to be small.

The study point is taken as $48.06^{\circ} \mathrm{W}$ and $84.86^{\circ} \mathrm{S}$, the same as taken Meng et al. [8]. The brightness temperature is $66.25,86.99,53.70$ and $69.93 \mathrm{~K}$ in $3,7.8,19.35$ and $37 \mathrm{GHz}$ respectively on $6^{\text {th }}$ May, 2008 . On $6^{\text {th }}$ July 2008, the corresponding temperatures of the same point are $66.47,91.79,55.47$ and $70.85 \mathrm{~K}$. The brightness temperature is very low at selected point 69.93 and $70.85 \mathrm{~K}$, at $37 \mathrm{GHz}$, where the penetration depth is low. The difference in the two observation time is only $0.92 \mathrm{~K}$. Thus the selected point matches the selection criteria.

It is known that the volume water ice content is directly related with microwave brightness temperature. The surface and deep physical temperature are taken as 78 and $134 \mathrm{~K}$ respectively [8]. The permittivity of the regolith with water ice can be obtained by Odelevsky model, where permittivity of lunar soil stimulant JSC-1A is $2.67+8.064 \mathrm{E}-07 \mathrm{i}$ [17]. The permittivity of the pure ice is taken as $4.01+6.53 \mathrm{i}$ [17]. The relation between volume water ice content and the brightness temperature can be obtained by radiative transfer equation and Odelevsky model. At $37 \mathrm{GHz}$, the measured brightness temperature at the selected point is 69.93 $\mathrm{K}$.

Fig. 3 shows the relation between brightness temperature and volume ice content obtained using the permittivity of the JSC-1A and pure ice as mentioned above. From Fig.3, it can be observed that the ice content at the study point is about $2.943 \%$, where $\mathrm{d}_{1}=0.09 \mathrm{~m}$ and $\mathrm{d}_{2}=9.91 \mathrm{~m}$. 


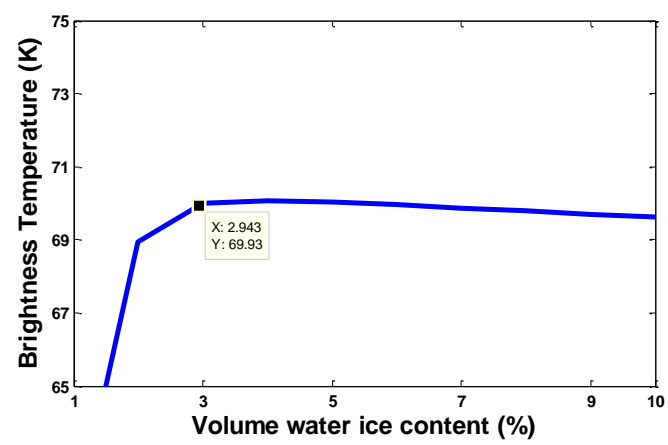

Figure 3: Relation between brightness temperature and ice content.

Meng and his colleagues [8] have shown that the volumetric water ice content at the study point is about $2.83 \%$.

\section{Conclusion}

In this article, we have revisited the water ice content in the Cabeus crater. We have used the measured data of lunar soil stimulant JSC-1A and the pure ice, and Odelevsky model to derive the effective permittivity of the mixture. The microwave brightness temperature has been simulated using two layer model of the lunar surface. The data from Chang'e-1 have been used at the study point. The results indicate that there is a possibility of water ice mixed with the regolith in the upper most layer whose depth is about $9 \mathrm{~cm}$ and the volume water ice content has been estimated as $2.943 \%$.

\section{References}

[1]. Arnold J R., "Ice in the lunar polar regions", J Geophys Res, 1979, 84:5659-5668.

[2]. Watson K, Murray B C, Brown H. "The behavior of volatiles on the lunar surface" J Geophys Res, 1961, 66: 3033-3045.

[3]. Nozette S, Lichtenberg C L, Spudis P, et al. "The Clementine bistatic radar experiment” Science, 1996, 274: 1495-1498.

[4]. Spudis, P., Ice on the Moon, The Space Review, November 6, 2006.

[5]. Feldman W C, Maurice S, Binder A B, et al. "Fluxes of fast and epithermal neutrons from lunar prospector: Evidence for water ice at the lunar Poles " Science, 1998, 281: 1496-1500.

[6]. Hodges R. Ice in the lunar polar regions revisited. J Geophys Res, 2002, 107:5011-5017.

[7]. Zhiguo Meng "Research on the Distribution and Content of Water Ice in Lunar Pole Regions Using Clementine UVVIS Data", Journal of Earth Science, Vol. 22, No. 5, p. 595-600, October 2011.

[8]. Meng ZhiGuo1, Chen \& Cui TengFeil,"Research on water ice content in Cabeus crater using the data from the microwave radiometer onboard Chang'e-1 satellite", Science China, December 2010 Vol.53 No.12: 2172-2178.

[9]. Feng Jianqing, et al, "Review on physical models of lunar brightness temperature" Chin.J.Geochem.,2009, Volume 29, 204-211.

[10]. Shkuratov Y G, Bondarenko N V. "Regolith layer thickness mapping of the moon by radar and optical data", Icarus, 2001, 149: 329-338.

[11]. Jin Y Q, Yan, et al. "Simulation of brightness temperature from the lunar surface using multi-channels microwave radiometers", Chin J Radio Sci, 2003, 18:477-486.

[12]. Meng Z G, Chen S B, Li C, et al. Review on retrieval of lunar regolith thickness by active and passive microwave measurements Global Geology, 2008, 11: 102-109.

[13]. Wang Z Z, Li Y, Jiang J S, et al. Lunar surface dielectric constant, regolith thickness and helium-3 abundance distributions retrieved from microwave brightness temperatures of CE-1 Lunar Microwave Sounder. Sci China Earth Sci, 2010, 53: 1365-1378.

[14]. Online, $10^{\text {th }}$ May 2014. Available: http://www.nasa.gov/mission_pages/LRO/multimedia//roimages/lroc_20091117_cabeus.html.

[15]. Shutko A M, Reutov E M. Mixture formulas applied in estimation ofdielectric radiative characteristics of soils and grounds at microwave frequencies. IEEE Trans Geosci Remote Sens, 1982, 20: 29-32.

[16]. Heiken et al. Lunar sourcebook: a user's guide to the moon. Cambridge:Cambridge University Press, 1991.

[17]. Jayesh Pabari, "Investigation of Wireless Sensor Network for Exploration of Lunar Ice: An in-situ approach towards lunar surface science", LAP Lambert Academic Publishing, 22-Oct-2013. 